\title{
Utilisation du glucose et du cellobiose par trois souches de Fibrobacter succinogenes
}

\author{
G Gaudet ${ }^{1}$, KJ Cheng² \\ 1 Laboratoire de Microbiologie, INRA, Theix, 63122 Ceyrat, France; \\ ${ }^{2}$ Agriculture Canada Research Station Lethbridge, Alberta, Canada T1J4B1
}

\begin{abstract}
Summary - F succinogenes strains $S 85,128$ and 095 were compared with respect to their growth using glucose and/or cellobiose as the carbon and energy substrate(s) and their capacities to degrade cellulose. The growth rate of F succinogenes strain S85 was the same using glucose or cellobiose, whereas the growth rates of strains 128 and 095 were about thrice the rate when using cellobiose. Strain S85 could simultaneously use glucose and cellobiose, while strains 128 and 095 tended to use preferentially glucose then cellobiose in a mixture of the 2 sugars. Their capacities to degrade cellulose were equivalent.
\end{abstract}

Introduction - Dans cette étude, trois souches d'une même espèce de bactérie cellulolytique du rumen $F$ succinogenes (ex Bacteroides succinogenes) ont été utilisées : $\mathbf{S} 85$ considérée comme la souche type de l'espèce (Montgomery et al, 1988), 128 et 095 isolées récemment, respectivement de contenu de rumen de buffle et de taureau, dans le laboratoire de KJ Cheng. La souche $S 85$ a été isolée et identifiée il y a plus de 30 ans (Hungate, 1950) puis utilisée depuis dans de nombreuses études tant in vivo que in vitro. Les multiples transferts nécessités pour sa conservation peuvent avoir provoqué des modifications physiologiques. Le but de ce travail est de comparer deux caractéristiques physiologiques de ces souches : la dégradation in vitro de la cellulose ef l'utilisation du glucose et du cellubiose, sucres produits lors de la cellulolyse.

Matériel et Méthodes - F succinogenes est cultivé sur milieu de Bryant et Burkey (1953) contenant $30 \%$ de jus de rumen. La croissance est suivie par la lecture de l'absorbance à 600 $\mathrm{nm}$. Les dosages de glucose sont effectués par la méthode standardisée de dosage du glucose UV-Boehringer. La dégradation de la cellulose de papier filtre est mesurée par gravimétrie.

Résultats et Discussion - La croissance de $F$ succinogenes, souche $S 85$ a été identique que la source de carbone du milieu soit du glucose ou du cellobiose, par contre les souches 128 et 095 se sont développées environ trois à quatre fois plus vite sur glucose que sur cellobiose (fig 1). L'addition de glucose ou de cellobiose en cours de culture respectivement sur cellobiose ou sur glucose, a provoqué une augmentation momentanée du taux de croissance chez la souche S85. Pour les souches 128 et 095, l'addition de glucose en cours de croissance sur cellobiose a provoqué l'augmentation définitive du taux de croissance jusqu'à la valeur habituellement mesurée sur glucose, l'addition de cellobiose en cours de croissance sur glucose a été sans effet.

Lors de la culture sur le mélange des. sucres, la consommation de glucose a été réduite, pour une augmentation équivalente de la population bactérienne (fig 2). 


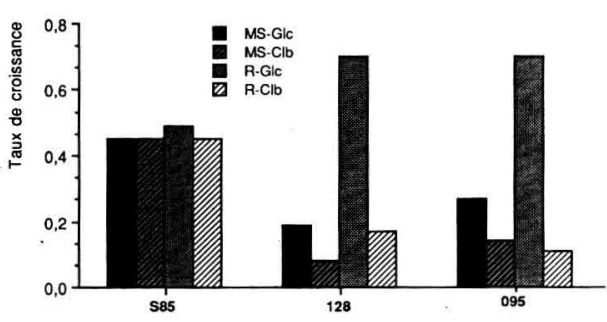

Fig 1. F succinogenes: taux de croissance comparé des trois souches S85, 128, 095, en fonction de la composition du milieu. MS = milieu synthétique; $R=$ milieu à $30 \%$ de jus de rumen; $\mathrm{Glc}=$ glucose $; \mathrm{Clb}=$ cellobiose .

La diminution de la consommation de glucose par rapport à la culture sur glucose seul est d'environ un tiers pour la souche 128 , d'un quart pour la souche 095 et de plus de la moitié pour la souche S85. Alors que les souches 128 et 095 ont montré une préférence nette pour le glucose, la souche S85 utilise simultanément les deux sucres.

In vitro, les trois souches ont dégradé la cellulose de la même manière soit 20 à 25 $\mathrm{mg}$ de cellulose de papier filtre digéré en $5 \mathrm{j}$ de culture dans $10 \mathrm{ml}$ de milieu.

II est classiquement admis que dans un mélange de sucre le glucose est préférentiellement voire exclusivement utilisé par les bactéries. L'utilisation simultanée du glucose et du cellobiose par la souche S85 peut résulter soit de la perte d'un mécanisme de régulation soit de l'existence d'une physiologie propre à cette souche.

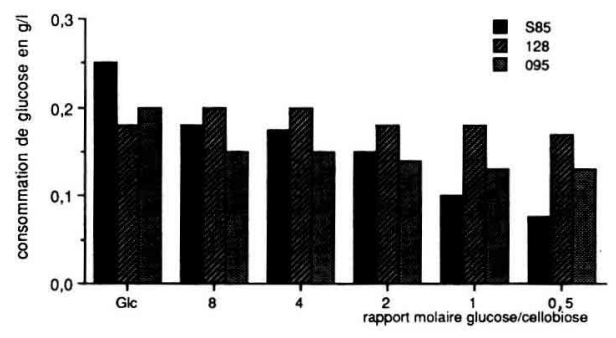

Fig 2. F succinogenes: consommation de glucose (en g/l) pour une augmentation de 0,2 unités DO en fonction des concentrations relatives en glucose et cellobiose (exprimées par le rapport molaire glucose/cellobiose). Glc = glucose seul.

Le mode d'utilisation de ces sucres, produits terminaux de la cellulolyse extracellulaire, capables d'entrer dans la cellule pour y être fermentés, conditionne l'utilisation de la cellulose comme source d'énergie pour la bactérie. La capacité à dégrader la cellulose in vitro ne s'est pas altérée au cours du temps pour la souche $S 85$, il y a donc peu de chance que le mode d'utilisation des sucres se soit modifié. Ceci laisse supposer l'existence d'une physiologie propre à la souche $S 85$ et nous incite à n'extrapoler qu'avec précaution les résultats obtenus en travaillant avec la souche S85 de l'espèce $F$ succinogenes.

Bryant MP, Burkey LA (1953) J Dairy Sci 36, 205-217

Hungate RE (1950) Bacteriol Rev 14, 1-49

Montgomery L, Flescher B, Stahl D (1988) Int J Syst Bacteriol 38, 430-435 